\title{
Desarrollo y validación de la Escala de Percepción de la Infidelidad en las Redes Sociales
}

\author{
Development and Validation of the Perception of Infidelity in \\ Social Networks Scale
}

\author{
Gladiminet López-Figueroa * 1 , Juan Aníbal González-Rivera ${ }^{2}$ \\ 1 - Carlos Albizu University, San Juan, Puerto Rico. \\ 2 - Ponce Health Sciences University, San Juan University Center, Puerto Rico.
}

Introducción

Marco Teórico

Método

Resultados

Discusión

Referencias

Recibido: 05/07/19 Revisado: 12/08/19 Aceptado: 03/09/19

\section{Resumen}

El objetivo principal de esta investigación fue desarrollar y validar la Escala de Percepción de la Infidelidad en las Redes Sociales (EPIRS) en una muestra de adultos puertorriqueños. Un total de 300 puertorriqueños participaron en este estudio de carácter exploratorio y psicométrico. La muestra fue seleccionada por disponibilidad. Los resultados confirmaron que la escala posee una estructura unidimensional. Un total de 12 ítems cumplieron con los criterios de discriminación y las cargas factoriales apropiados. El índice de confiabilidad alfa de Cronbach de la versión final fue .98. Estos resultados sugieren que la EPIRS tiene el potencial para medir este constructo en adultos puertorriqueños. Además, la escala permitirá el avance de nuevas investigaciones sobre la infidelidad cibernética en parejas puertorriqueñas y latinoamericanas.

Palabras clave: infidelidad, redes sociales, relaciones románticas, propiedades psicométricas, validación

\begin{abstract}
The purpose of this study was to develop and validate the Perception of Infidelity in Social Networks Scale. A total of 300 Puerto Rican adults participated in this psychometric study. The sample was selected by availability. The results confirmed that the scale has a one-dimensional structure. A total of 12 items complied with the criteria of discrimination and presented appropriate factor loadings. The reliability index for the final version was .98 (Cronbach's alpha). These results suggest that the Perception of Infidelity in Social Networks Scale has the potential to measure this construct among Puerto Rican adults. Likewise, the scale will advance further research of cyber-infidelity in Puerto Rican and Latin American couples.
\end{abstract}

Key words: infidelity, romantic relationships, social networks, psychometric properties, validation

\footnotetext{
* Correspondencia a: Dra. Gladiminet López Figueroa, 500 West Main Suite 215, Bayamón, Puerto Rico, 00961. Tel.: 011787 604 4999. E-mail: gladiminet@hotmail.com. Para permiso de uso y descargar el instrumento, visite www.juananibalgonzalez.com

Cómo citar este artículo: López-Figueroa, G., \& González-Rivera, J. A. (2019). Desarrollo y validación de la Escala de Percepción de la Infidelidad en las Redes Sociales. Revista Evaluar, 19(3), 54-67. Recuperado de https://revistas.unc.edu.ar/index.php/revaluar

Nota de autor: Los autores expresan que no hubo conflictos de intereses al redactar el manuscrito.
} 


\section{Introducción}

El internet ha tenido una gran influencia en la manera en la que se relacionan las personas consigo mismas y con los demás (Gackenbach \& von Stackelberg, 2007). Es infinita la cantidad de posibilidades que tienen las personas detrás de un teclado. Este tipo de comunicaciones eliminan las limitaciones geográficas, las impuestas por la vestimenta, el aspecto, la edad, el tiempo disponible y el clima, e incluso eliminan el riesgo de contagio de enfermedades. El uso de la tecnología abrió paso para que los psicólogos y profesionales de la salud mental incorporaran este fenómeno dentro de sus prácticas de servicios psicológicos. A su vez, provocó que se comenzara a estudiar la conducta en línea (conocida en inglés como online behavior), y cómo esta conducta difiere del comportamiento real de las personas (conocido en inglés como offline behavior).

Se ha encontrado que las motivaciones, los deseos, las necesidades y las habilidades que guían una conducta en línea no son iguales a las que guían una conducta real (Attrill, 2015). Por ejemplo, existen factores del lenguaje expresivo que se tornan ausentes o invisibles en una conversación en línea. La manera en que las personas hablan, su tono de voz, ritmo, entonación, volumen, fluidez, entre otros, son señales del uso del lenguaje expresivo que no se presentan en una conversación en línea. Este fenómeno de comunicación puede llevar a malos entendidos y las partes implicadas en la conversación tienden a interpretar individualmente el tono del mensaje. Sin embargo, las nuevas plataformas que permiten el uso de videoconferencias, como Skype, WhatsApp y Snapchat, favorecen el lenguaje expresivo y minimizan la posibilidad de malos entendidos.

Por otra parte, estudios revelan que las personas invierten uno de cada cuatro minutos en una red social, por lo que se infiere que estas utilizan los medios de comunicación social para establecer nuevas relaciones y para conectar y mantener relaciones de intimidad (Wasserman, 2015). De esta forma, las redes sociales se han convertido en un medio para establecer relaciones de interacción social virtual y, así, la infidelidad cibernética, que es una manera ideal para esconder estas actividades a la pareja, va adquiriendo terreno ya que no existe evidencia obvia del encuentro (Schneider, 2000).

\section{Marco Teórico}

La palabra infidelidad proviene del latín infidelitas formado por in (negación) y fidelitas (fidelidad). Así, se entiende que la palabra significa el incumplimiento de la fidelidad (Varela-Maceda, 2014). Según González-Galarza, Martínez-Taboas y Martínez-Ortiz (2009), la infidelidad se define como el incumplimiento al supuesto fundamental de exclusividad en aspectos de intimidad emocional o sexual de una pareja romántica. Otros autores la definen como una violación a la promesa de no tener relaciones íntimas con otras personas sin el consentimiento del otro miembro de la pareja (Pérez-Baquero, Ruiz-Santos, \& Parra-Ocampo, 2014). Por otra parte, para Berman y Frazier (2005), la infidelidad es una relación secreta de carácter sexual o emocional con otra persona que no se considera la pareja primaria y que podría resultar inaceptable para la pareja primaria si se enterase de la relación extradiádica. Para Glass (2002), la infidelidad se refiere a una conducta secreta de tipo sexual, emocional o romántica que viola las normas de exclusividad en una relación romántica.

Investigadores como Bernard (1974), Pittman y Wagers (2005) y Romero-Palencia (2007) han enfatizado una serie de cualidades que definen el constructo de infidelidad, aunque sus 
componentes no sean siempre consistentes. Por ejemplo, Bernard (1974), indica que cuando un individuo falla en demostrar amor, honor y apoyo a su pareja, estaría violando los votos de su relación romántica $\mathrm{y}$, por lo tanto, estaría siendo infiel. En cambio, Pittman y Wagers (2005) plantean que el signo primordial de la infidelidad es la secretividad y el encubrimiento de la conducta extradiádica. Asimismo, Romero-Palencia (2007) postula una definición de infidelidad basada en los siguientes tres aspectos: a) la conducta extradiádica no es permitida por la pareja romántica, b) la conducta ocurre fuera de la relación primaria, y c) ocurre una conducta extradiádica concreta, como podría ser el coito, el coqueteo, entre otros.

Un estudio encontró que el 91\% de los adultos estadounidenses consideran las relaciones de infidelidad extramarital moralmente incorrectas (Munsch, 2015). Aun cuando es considerada moralmente incorrecta, la infidelidad continúa siendo un asunto ordinario observado en las relaciones de pareja (Allens et al., 2005). Diversos estudios realizados recientemente en Estados Unidos indican que entre un 26\% y un 50\% de los hombres y entre un $27 \%$ y un $35 \%$ de las mujeres tendrán por lo menos una relación extramarital de tipo sexual (Wasserman, 2015). En un estudio de Whisman y Snyder (2007) se utilizó como fuente de datos una muestra de 4884 mujeres casadas que tenían entre 15 y 44 años de edad. Se les preguntó si habían tenido sexo extramarital en el último año y un 6\% de las mujeres admitió haber tenido esta experiencia. Por otra parte, un estudio reciente de Tafoya y Spitzberg (2007) destacó que el $34 \%$ de los hombres casados y el $24 \%$ de las mujeres casadas habían tenido una relación sexual extramarital.

Con frecuencia, se destaca que la infidelidad puede ocurrir de manera emocional y sexual. La infidelidad sexual ocurre cuando un individuo tiene una involucración sexual fuera de su rela- ción primaria con o sin el consentimiento de su pareja (Wasserman, 2015). Según Mark, Janssen y Milhausen (2011), la infidelidad sexual es el intercambio sexual extradiádico dentro de una relación monógama. Por otra parte, la infidelidad emocional ocurre cuando una persona con pareja emplea su tiempo, atención, romanticismo y expresiones de afecto con una persona que no es su pareja primaria (Shackelford, LeBlanc, \& Drass, 2000). Sin embargo, cuando ocurre una infidelidad que incorpora el área emocional y el área sexual, se considera una infidelidad compuesta (Hall \& Fincham, 2006). Investigadores han descubierto que la infidelidad también puede ocurrir en otras categorías más generales como las de solo una noche, las relaciones a largo plazo, las relaciones a través de internet y las relaciones que ocurren en el lugar de trabajo (Blow \& Hartnett, 2005).

Para efectos de esta investigación, se contemplará únicamente la infidelidad en las redes sociales, definida como un proceso en el que una persona involucrada en una relación marital o de compromiso utiliza un equipo de contacto interactivo en conversaciones electrónicas producidas a través de las redes sociales (por ejemplo, Facebook, Instagram, Twitter, WhatsApp, Snapchat, Kik, salas de chat variadas, correos electrónicos y plataformas de citas) para tener contacto emocional y/o sexual con una persona que no sea la pareja primaria. El contacto por lo general se caracteriza por la secretividad y la violación de los principios de monogamia, fidelidad sexual y compromiso (Wasserman, 2015).

Otros autores han definido la infidelidad concentrándose en conductas específicas como: tener una cita, coquetear y tener sexo con una persona que no sea la pareja primaria (Luo, Cartun, \& Snider, 2010). Por otra parte, los investigadores Wilson, Mattingly, Clark, Weidler y Bequette (2011), al desarrollar una escala para medir el 
grado de percepción de ciertas conductas como la infidelidad, encontraron que las actividades catalogadas como infidelidad se dividen en tres categorías: conductas ambiguas (salir con una persona, hablar por teléfono o conversar a través de internet, etc.), conductas engañosas (mentir o no compartir cierta información a la pareja, entre otros) y conductas explícitas (tener sexo, tener una cita con una persona que no sea su pareja primaria, etc.).

Las personas que indican haber sido infieles, informan momentos placenteros de índole sexual, emocional y personal, sensaciones frescas, vibrantes y placenteras y experiencias de variedad sexual y excitación (Lawson, 1988). En los casos en los que se practica una relación abierta, la infidelidad puede añadir una nueva dimensión a la relación y a la vida sexual (Allan, 2004).

En cambio, se han presentado algunos riesgos relacionados a la práctica de la infidelidad, ya que se considera una de las causas más frecuentes de divorcio en la pareja (Cano \& O'Leary, 2000). A su vez, personas que han descubierto que su pareja les había sido infiel, informaron reacciones emocionales intensas como, por ejemplo, celos, coraje y repugnancia (Becker, Sagarin, Guadagno, Millevoi, \& Nicastle, 2004). El descubrimiento de una infidelidad está asociado con una reducción en el bienestar psicológico, incluido un aumento en la incidencia de depresión, ansiedad y en la reducción de la autoestima (Buunk, 1995). Igualmente, individuos que practican la infidelidad han reportado tener sentimientos de culpa, remordimiento, depresión, miedo de herir a su pareja y desaprobación de familiares y amigos luego de la transgresión en la relación (Allen \& Baucom, 2006).

Cada vez más seguido, las personas en una relación marital o en relaciones de compromiso desarrollan relaciones íntimas en las redes sociales sin el consentimiento de sus parejas primarias, lo que es una brecha que atenta contra los votos de fidelidad sexual, fidelidad emocional, monogamia y compromiso que realiza una pareja. Incurrir en esta conducta en línea es considerado como infidelidad cibernética. Una posible explicación para la ocurrencia de la infidelidad cibernética podría radicar en el resultado natural de un número extraordinario de personas en línea que se sienten insatisfechas en aspectos sexuales y/o emocionales con sus parejas en la vida real (Wasserman, 2015).

A estos efectos, este estudio pretende desarrollar y validar la Escala de Percepción de la Infidelidad en las Redes Sociales (EPIRS) en una muestra de adultos puertorriqueños. La escala tiene como objetivo lograr discriminar las conductas que son consideradas como infidelidad en las redes sociales de las que no lo son, para así tener una medida de este fenómeno social. También, este estudio aportará resultados significativos en términos de diferencias generacionales, ya que se espera determinar si existe algún tipo de variación o discrepancia del concepto de infidelidad en las redes sociales en función de la edad.

\section{Método \\ Diseño}

Esta investigación tiene un diseño de estudio instrumental (Ato, López-García, \& Benavente, 2013; Montero \& León, 2007). En esta se desarrolló y validó preliminarmente la EPIRS en una muestra de adultos puertorriqueños. Los autores buscaban desarrollar una medida justa para diferenciar la percepción de las personas sobre las conductas que son consideradas como infidelidad en las redes sociales de las que no lo son. 


\section{Participantes}

Se trabajó con una muestra no probabilística constituida por 300 participantes mayores de 21 años y residentes de Puerto Rico. Para determinar el tamaño de la muestra, seguimos las recomendaciones de Comrey y Lee (1992, p. 217), quienes sugieren que "la adecuación del tamaño muestral puede ser evaluada con la siguiente escala: 50 muy deficiente; 100 - deficiente; 200 - aceptable; 300 - bueno; 500 - muy bueno, 1000 o más - excelente". En la Tabla 1 se presentan los datos sociodemográficos de los participantes. Para participar en este estudio, se establecieron los siguientes criterios de inclusión: (1) ser mayor de 21 años de edad y (2) ser residente en Puerto Rico.

\section{Tabla 1}

Datos sociodemográficos de la muestra de directivos.

\begin{tabular}{lcc}
\hline \multicolumn{1}{c}{ Variables } & $\boldsymbol{f}$ & $\mathbf{\%}$ \\
\hline Sexo & 196 & 65.0 \\
Masculino & 104 & 35.0 \\
Femenino & & \\
Edad & 143 & 47.7 \\
21 a 30 años & 68 & 22.7 \\
31 a 40 años & 36 & 12.0 \\
41 a 50 años & 23 & 7.7 \\
51 a 60 años & 30 & 10.0 \\
60 a 65 años & & \\
Preparación académica & 152 & 50.7 \\
Bachillerato & 63 & 21.0 \\
Maestría & 19 & 6.3 \\
Doctorado & 6 & 2.0 \\
Post doctorado & 60 & 20.0 \\
Otros & & \\
Estado Civil & 180 & 60.0 \\
Soltero/a & 105 & 35.0 \\
Casado/a & 15 & 5.0 \\
Otros & & \\
\hline Nota $\mathrm{N}=300$ &
\end{tabular}

Nota. $N=300$.

\section{Instrumentos}

Cuestionario de Datos Generales. Para identificar las características sociodemográficas de la muestra, se desarrolló un cuestionario de datos generales, que recogía información importante en cuanto a la edad, sexo, estado civil y preparación académica.

Escala de Percepción de la Infidelidad en las Redes Sociales (EPIRS, versión preliminar). Este instrumento fue desarrollado por los investigadores luego de una extensa revisión de literatura con el propósito de desarrollar una medida justa para diferenciar la percepción de las personas sobre las conductas que son consideradas infidelidad en las redes sociales de las que no lo son. La versión original del instrumento estuvo constituida por 60 premisas que fueron sometidas a la opinión de 8 jueces expertos (doctores en psicología clínica) con el objetivo de identificar si los ítems de los instrumentos eran pertinentes a la infidelidad en las redes sociales (Método de Lawshe). Para rechazar o retener los ítems se utilizó el contentvalidityratio (CVR). Para interpretar los resultados, utilizamos los valores críticos recalculados por Wilson, Pan y Schumsky (2012). De acuerdo con estos autores, el valor mínimo requerido para 8 jueces según la tabla de Schipper es .75 para aceptar un ítem como esencial. Luego de efectuar el cálculo, identificamos 21 ítems con valores inferiores a .75 por lo cual fueron eliminados, lo que dejó el instrumento preliminar en 39 ítems, que fueron administrados en el presente estudio. El instrumento tiene una escala de respuesta tipo Likert de cuatro puntos: 1 (Totalmente en desacuerdo), 2 (En desacuerdo), 3 (De acuerdo), y 4 (Totalmente de acuerdo). El puntaje más bajo que se puede obtener en la versión preliminar es 39 y el más alto es 156. 


\section{Procedimientos}

La recopilación de datos se llevó a cabo de forma presencial en distintos centros comunitarios de la zona metropolitana, la zona central y la zona este de Puerto Rico. Para proteger y garantizar los derechos de los participantes, utilizamos una hoja de consentimiento informado en la que se notificaba lo siguiente: (a) el propósito del estudio, (b) la naturaleza voluntaria del estudio, (c) los posibles riesgos y beneficios, (d) el derecho del participante a retirarse del estudio en cualquier momento, (e) la institución de procedencia y (f) los datos de identificación y contacto de los investigadores. El consentimiento informado y el instrumento no estaban pareados, de esta forma se mantuvo en el anonimato y la privacidad. Para ello, se proveyó a los participantes un sobre de manila donde podían depositar los documentos de participación una vez completados. El consentimiento informado se entregó individualmente una vez completado, y se guardaba en una carpeta que contenía únicamente los consentimientos informados de los participantes. Una vez recopilados los datos, estos se ingresaron en una base de datos utilizando el sistema para análisis estadísticos IBM SPSS versión 24.0 (IBM Corporation, 2016). En este programa se realizaron análisis descriptivos de la muestra, análisis factorial exploratorio, análisis de discriminación de ítems, análisis de confiabilidad y análisis de comparación.

Para el análisis factorial exploratorio se utilizó el método de extracción de mínimos cuadrados no ponderados con rotación oblicua para identificar las variables latentes que subyacen en los ítems. Se identificaron aquellos factores que expliquen 5\% o más de la varianza como sugiere Hatcher (1994). Se utilizó este procedimiento de ajuste por dos razones principales: (1) el método de extracción de mínimos cuadrados no ponde- rados es el más recomendado actualmente para trabajar con muestras pequeñas -incluso cuando el número de variables es elevado-, especialmente si el número de factores a retener es pequeño (Jung, 2013) y evita la aparición de casos Heywood (saturaciones mayores que la unidad y varianzas de error negativas), más frecuentes con otros métodos de estimación (Lloret-Segura, Ferreres-Traver, Hernández-Baeza, \& Tomás-Marco, 2014); y (2) la rotación oblicua es más precisa y provee más información que la rotación octogonal (Schmitt, 2011). Como criterios de aceptación, consideramos aquellos ítems con una carga factorial mayor a .50 en un solo factor (Stevens, 2002). Para conocer la capacidad de discriminación de los ítems de los factores se calculó el índice de correlación ítem total (rbis). Para conocer el grado de confiabilidad de la escala, se calcularon el coeficiente alfa de Cronbach, alfa de Cronbach estandarizado y el coeficiente de división en mitades de Spearman-Brown. Field (2013) y Kline (2000) indican que índices mayores a .70 son aceptables.

\section{Resultados \\ Análisis descriptivos de los resultados}

Con la intención de conocer la percepción de los participantes sobre la infidelidad en las redes sociales y sobre cuáles conductas específicas son consideradas engañosas, se organizaron descriptivamente (frecuencia y porcentaje) las respuestas del cuestionario administrado. Con estos datos, se obtendrá un cuadro preliminar sobre aquellas conductas que son consideradas como infidelidad en las redes sociales por los participantes. La Tabla 2 muestra los tipos de conductas asociadas generalmente con la infidelidad en las redes sociales y la cantidad de personas de la muestra que las considera realmente como infidelidad. 
Tabla 2. Distribución de las conductas consideradas como infidelidad en las redes sociales.

\begin{tabular}{|c|c|c|}
\hline Conductas que realiza la pareja & f & $\%$ \\
\hline $\begin{array}{l}\text { 1. Ver material sexual a solas por } \\
\text { internet. }\end{array}$ & 153 & 51 \\
\hline $\begin{array}{l}\text { 2. Ver material sexual con otra persona } \\
\text { por internet que no sea yo. }\end{array}$ & 224 & 74.7 \\
\hline $\begin{array}{l}\text { 3. Enviar fotos a otra persona por las } \\
\text { redes sociales. }\end{array}$ & 184 & 71.3 \\
\hline $\begin{array}{l}\text { 4. Enviar fotos desnudo/a a otra perso- } \\
\text { na por las redes sociales. }\end{array}$ & 250 & 83.4 \\
\hline $\begin{array}{l}\text { 5. Enviar fotos semi-desnudo/a a otra } \\
\text { persona por las redes sociales. }\end{array}$ & 245 & 81.6 \\
\hline $\begin{array}{l}\text { 6. Enviar fotos provocativas a otra } \\
\text { persona por las redes sociales. }\end{array}$ & 240 & 80.0 \\
\hline $\begin{array}{l}\text { 7. Conectarse diariamente a portales } \\
\text { sexuales. }\end{array}$ & 188 & 62.6 \\
\hline $\begin{array}{l}\text { 8. Conectarse ocasionalmente a porta- } \\
\text { les sexuales. }\end{array}$ & 157 & 52.4 \\
\hline $\begin{array}{l}\text { 9. Utilizar las redes sociales para tener } \\
\text { conversaciones eróticas. }\end{array}$ & 244 & 81.3 \\
\hline $\begin{array}{l}\text { 10. Utilizar las redes sociales para tener } \\
\text { conversaciones "calientes" con otra } \\
\text { persona. }\end{array}$ & 250 & 83.3 \\
\hline $\begin{array}{l}\text { 11. Tener una cuenta creada en un por- } \\
\text { tal de índole sexual. }\end{array}$ & 229 & 76.3 \\
\hline $\begin{array}{l}\text { 12. Conversar diariamente con una } \\
\text { persona que conoció por alguna de las } \\
\text { redes sociales. }\end{array}$ & 198 & 66.0 \\
\hline $\begin{array}{l}\text { 13. Conversar ocasionalmente con una } \\
\text { persona que conoció por alguna de las } \\
\text { redes sociales. }\end{array}$ & 156 & 52.0 \\
\hline $\begin{array}{l}\text { 14. Seguir en las redes sociales cuentas } \\
\text { de personas que le parezcan atractivas. }\end{array}$ & 150 & 50.0 \\
\hline $\begin{array}{l}\text { 15. Masturbarse a solas viendo portales } \\
\text { sexuales en internet. }\end{array}$ & 158 & 52.6 \\
\hline $\begin{array}{l}\text { 16. Negarse a darme las contraseñas de } \\
\text { sus redes sociales. }\end{array}$ & 156 & 52.0 \\
\hline $\begin{array}{l}\text { 17. No permitirme utilizar sus aparatos } \\
\text { electrónicos. }\end{array}$ & 164 & 54.6 \\
\hline $\begin{array}{l}\text { 18. Abrir una cuenta en las redes socia- } \\
\text { les sin que yo tenga conocimiento. }\end{array}$ & 170 & 56.7 \\
\hline $\begin{array}{l}\text { 19. Tener sexo cibernético con otra } \\
\text { persona en las redes. }\end{array}$ & 255 & 85.0 \\
\hline $\begin{array}{l}\text { 20. No decir con quién comparte en las } \\
\text { redes sociales. }\end{array}$ & 165 & 55.0 \\
\hline $\begin{array}{l}\text { 21. Recibir material sexual a través de } \\
\text { las redes sociales. }\end{array}$ & 193 & 64.4 \\
\hline
\end{tabular}

22. Enviar fotos de sus genitales a otra

258

86.0 persona.

23. Buscar apoyo emocional de otras

181 personas en las redes.

24.Enviar regalos a otra persona a través de internet.

25. Recibir regalos de otra persona a través de internet.

26. Tener conversaciones en vivo con otra persona.

27. Subir fotos provocativas de sí mismo/a en las redes.

28. Conversar con otra persona en las

79.6 redes sociales y que comparta cosas más íntimas y constantes que las que comparte conmigo.

29. Guardar fotos de otra persona en las

208 redes sociales.

30. Cerrar rápidamente las aplicaciones

de las redes sociales cuando yo me acerco.

31. Mentir sobre sus actividades perso- $\quad 232 \quad 77.3$ nales en internet.

32. Incomodarse al compartir conmigo

210

el contenido de sus redes.

33. Tener en las redes sociales a sus

ex-parejas.

34. Tener en las redes sociales perso-

nas con las cuales tuvo algún tipo de contacto sexual.

35. Tenerme bloqueado de sus redes

232

77.3 sociales.

36. No tenerme en sus redes sociales.

212

70.7

37. No tener en sus redes sociales su

171 verdadero estado civil.

38. No tener fotos conmigo en las redes

166

55.3 sociales.

39. Borrar constantemente el historial

203

67.6 de los chats en las redes sociales.

$\overline{\text { Nota. La frecuencia representa la cantidad de personas de }}$ la muestra que considera la conducta como infidelidad. $\mathrm{N}=300$. 


\section{Análisis factorial exploratorio}

Se realizó un primer análisis factorial exploratorio utilizando el método de extracción de mínimos cuadrados no ponderados con rotación oblicua. Los resultados mostraron inicialmente una estructura de cuatro factores que explican el $75.34 \%$ de la varianza de los datos. De estos, solo dos factores explicaban $5 \%$ o más de la varianza. Al revisar las cargas factoriales de los 39 ítems, se identificaron siete ítems que no cumplieron con los requisitos de retención (carga factorial de .50 o más en un solo factor), por lo cual se eliminaron los ítems 14, 21, 27, 30, 34, 37 y 38.

Luego de eliminar estos siete ítems, se procedió nuevamente a hacer un análisis factorial con los 32 ítems restantes. Los resultados reflejaron tres factores que explicaban el $77.62 \%$ de la varianza. Nuevamente, solo dos factores explicaban $5 \%$ o más de la varianza. Al revisar las cargas factoriales de los 32 ítems, se observó que dos ítems (33 y 39 ) cargaban menos de .50 , por lo cual fueron eliminados. Se realizó por tercera ocasión un análisis factorial con los 30 ítems restantes. Nuevamente, los resultados reflejaron tres factores que explicaban el $78.75 \%$ de la varianza. Al revisar las cargas factoriales de los 30 ítems, se observó que el reactivo 32 cargaba más de .50 en dos factores, por lo cual fue eliminado. Además, se eliminaron todos los ítems que cargaron negativamente en uno de los factores $(3,12,13,23$, $24,25,26$ у 29).

Luego, se realizó nuevamente un análisis de factores con los 21 ítems. Los resultados reflejaron tres factores que explicaban el $80.75 \%$ de la varianza. No obstante, uno de los factores recogió ítems que podrían estar relacionados con asuntos de privacidad y espacio personal o conduc- tas celotípicas. En muchas ocasiones, este tipo de conductas están asociadas a violencia psicológica hacia la pareja. Otro de los factores recogió ítems asociados a la búsqueda de placer por parte de la pareja mediante la masturbación y la observación de material sexual para adultos estando a solas o en privado. Estos últimos dos factores, como quedaron constituidos, no forman parte del marco teórico de infidelidad, ya que explican mejor otros fenómenos asociados con las redes sociales. Por lo tanto, se decidió eliminar todos los ítems correspondientes a estos dos factores $(1,2,7,8,15,16$, 17,18 y 20). Por última vez, se realizó un análisis factorial con los 12 ítems restantes, con la hipótesis de que todos cargarían más de .50 en un solo factor, y así sucedió. Los resultados confirmaron que los ítems se agrupaban en un único factor que explicaba el $80.59 \%$ de la varianza. La Tabla 3 presenta las cargas factoriales de los ítems.

\section{Análisis de los ítems}

Con los 12 ítems restantes, se realizó un análisis de discriminación a través del índice de correlación ítem-total $\left(r_{\text {bis }}\right)$. Además, se calculó la varianza explicada de la escala total en los ítems. La Tabla 3 presenta los índices de discriminación de la versión final de la escala, los cuales están por encima del mínimo recomendado de .30 (Kline, 2005) y la varianza explicada por cada ítem. 
Tabla 3

Cargas factoriales de ítems, índices de discriminación y varianza explicada.

\begin{tabular}{|c|c|c|c|}
\hline Ítem & Factor 1 & $r_{\text {bis }}$ & $\mathbf{R}^{2}$ \\
\hline $\begin{array}{l}\text { 10. Que mi pareja utilice las redes sociales para tener conversaciones "calientes" con otra } \\
\text { persona. }\end{array}$ & .955 & 94 & 91 \\
\hline 19. Que mi pareja tenga sexo cibernético con otra persona en las redes sociales. & .933 & 92 & 93 \\
\hline 22. Que mi pareja envíe fotos de sus genitales a otra persona. & .929 & 92 & 93 \\
\hline $\begin{array}{l}\text { 5. Que mi pareja envíe una foto semi-desnudo/a de él/ella a otra persona a través de alguna } \\
\text { de las redes sociales. }\end{array}$ & .927 & 91 & 92 \\
\hline 9. Que mi pareja utilice las redes sociales para tener conversaciones eróticas. & .921 & 91 & 89 \\
\hline $\begin{array}{l}\text { 4. Que mi pareja envíe una foto desnudo/a de él/ella a otra persona a través de alguna de las } \\
\text { redes sociales. }\end{array}$ & .915 & 90 & 90 \\
\hline $\begin{array}{l}\text { 6. Que mi pareja envíe una foto provocativa de él/ella a otra persona a través de alguna de } \\
\text { las redes sociales. }\end{array}$ & .911 & 90 & 88 \\
\hline $\begin{array}{l}\text { 28. Que mi pareja converse con otra persona en las redes sociales y lo que comparta con } \\
\text { ella sea más íntimo y constante de aquello que comparte conmigo. }\end{array}$ & .856 & 85 & 74 \\
\hline 31. Que mi pareja mienta sobre sus actividades personales en internet. & .852 & 85 & 76 \\
\hline 35. Que mi pareja me tenga bloqueado/a de sus redes sociales. & .845 & 84 & 84 \\
\hline 11. Que mi pareja tenga una cuenta creada en un portal de índole sexual. & .840 & 83 & 71 \\
\hline 36. Que mi pareja no me tenga en sus redes sociales. & .754 & 75 & 78 \\
\hline
\end{tabular}

Nota. $r_{\text {bis }}=$ Indice de discriminación; $\mathrm{R}^{2}$ = Varianza explicada; Factor 1 = carga factorial.

\section{Análisis de Confiabilidad}

Los 12 ítems fueron sometidos a un análisis de consistencia interna para determinar el índice de confiabilidad de la escala. Para ello, se utilizaron dos métodos: el coeficiente alfa de Cronbach y división en mitades de Spearman-Brown. Los resultados mostraron un coeficiente alfa de .98 y de .98 para la prueba Spearman-Brown. En la Tabla 4 se presentan el alfa de Cronbach, el coeficiente de la prueba Spearman-Brown, la media y la desviación estándar de la escala.

\section{Análisis comparativo}

Posteriormente, se realizó un análisis de varianza para evaluar si existen diferencias por edad en cuanto a las conductas promedio consid- eradas como infidelidad en las redes sociales. Los resultados de la prueba Anova confirmaron que hay diferencias estadísticamente significativas en las medias por grupo de edad $\left[\mathrm{F}_{(4,295)}=4.036, p=\right.$ .003]. Para identificar entre qué grupo son las diferencias significativas, se realizó una prueba post hoc Games-Howell, ya que la prueba de Levene fue significativa (Field, 2013). El grupo de edad 60 en adelante $(\mathrm{M}=46.00)$ difiere estadísticamente con los otros grupos: 21-30 $(\mathrm{M}=37.58)$, 31-40 ( $\mathrm{M}=39.05)$, y 41-50 $(\mathrm{M}=41.22)$. No obstante, no difiere estadísticamente con el grupo 51-60 ( $\mathrm{M}=42.30)$. Los otros grupos no difieren estadísticamente entre ellos. A su vez, se realizó un análisis de correlación producto-momento de Pearson para analizar el grado de asociación entre la edad de los participantes y su percepción de la infidelidad en las redes sociales. Los resultados obtenidos sugieren una asociación positiva y baja entre la edad y percepción de la infidelidad en las redes sociales $\left[r_{(300)}=.23, p<.01\right]$. Este resulta- 
do sugiere que, a mayor rango de edad, mayores serán las conductas percibidas como infidelidad en las redes sociales.

\section{Discusión}

El propósito principal de este estudio fue desarrollar y validar la EPIRS en una muestra de adultos puertorriqueños. Para lograrlo, se realizaron varios análisis de carácter psicométrico que permitieron de manera sistemática la validación del instrumento. Los hallazgos confirmaron que la versión final del instrumento posee propiedades psicométricas adecuadas para medir la percepción de la infidelidad en las redes sociales desde un modelo unidimensional. A su vez, los índices de confiabilidad alcanzados respaldan estadísticamente la consistencia interna del instrumento final.

En términos teóricos, el análisis factorial exploratorio mostró la existencia de un solo factor subyacente en los ítems finales del instrumento, contrario al modelo teórico propuesto por Wilson et al. (2011), quienes sugieren tres dimensiones de conductas catalogadas como infidelidad: conductas ambiguas, conductas engañosas y conductas explícitas. No obstante, esta única dimensión recoge algunos aspectos de los propuestos por Wilson et al. (2011). Por ejemplo, los ítems 10, 19, 22, 5, 9, 4 y 6 representan conductas concretas típicamente asociadas a la infidelidad; los ítems 11,28 y 36 suponen conductas que no se asocian de forma clara con la infidelidad, pero en las que puede existir una posibilidad de traición; $y$, por último, los ítems 31 y 35 están relacionados con la mentira y el ocultamiento de información. Por consiguiente, la estructura unifactorial y las excelentes propiedades psicométricas obtenidas por la escala sugieren que la versión final del instrumento es lo suficientemente eficiente para cubrir varios aspectos de la percepción de la infidelidad en las redes sociales de una forma global.

El comportamiento de la escala mostró cuáles son aquellos aspectos que los puertorriqueños identifican como conductas asociadas a la infidelidad en las redes sociales. Específicamente, estas conductas son: (1) enviar fotos desnudas o provocativas a otra persona; (2) tener sexo cibernético con otra persona; (3) tener conversaciones "calientes" o eróticas con otra persona; (4) tener mayor comunicación o intimidad emocional con otra persona que con la pareja principal; (5) tener cuentas creadas en portales de índole sexual; (6) no permitir que la pareja tenga acceso a sus redes sociales y (7) mentir sobre las actividades personales que realiza en las redes sociales. Estas conductas se caracterizan por la secretividad y violan los principios de monogamia, fidelidad sexual y compromiso, típicamente aceptados por la sociedad (Wasserman, 2015). Por ejemplo, Munsch (2015) encontró que el 91\% de los estadounidenses considera este tipo de conductas como moralmente incorrectas.

En cuanto a la confiabilidad del instrumento, se obtuvieron índices superiores al mínimo recomendado por la literatura científica (DeVellis, 2017). Lo cual sugiere que la versión final de la escala es un instrumento estable, reproducible y consistente en la medida de la percepción de la infidelidad en las redes sociales. Es decir, la EPIRS cuenta con la confiabilidad necesaria para poder ser utilizada como instrumento de investigación en el contexto puertorriqueño. Por su parte, las correlaciones de cada ítem con la puntuación total manifiestan una consistencia interna notable. Esto sugiere que los ítems de la versión final discriminan adecuadamente y son capaces de diferenciar personas con distintas percepciones y opiniones sobre la infidelidad en las redes sociales.

En cuanto a la edad, los análisis confirmaron que existen diferencias estadísticamente sig- 
nificativas en las medias del grupo de edad 60 en adelante y los otros grupos de edad. Además, se encontró una correlación directa, moderada baja y estadísticamente significativa entre la edad y las conductas consideradas como infidelidad en las redes sociales. En Puerto Rico, parece ser que las personas de tercera edad son más severas en la clasificación de conductas asociadas a la infidelidad en las redes sociales. Según Docan-Morgan y Docan (2007), las personas más jóvenes perciben como prácticas cotidianas y típicas aquellas conductas que los más adultos clasificarían como infidelidad. A su vez, explican que las personas mayores tienden a tener límites y normas más rígidas dentro de sus relaciones de pareja. En síntesis, ciertas conductas asociadas a la infidelidad, principalmente las conductas ambiguas, podrían ser parte del comportamiento normativo de generaciones más jóvenes.

En términos prácticos, se demostró que la escala final puede ser utilizada para el desarrollo de nuevas investigaciones sobre el tema de la infidelidad en las redes sociales y sus posibles correlaciones con otras variables psicosociales. Sobre el uso del instrumento, debemos señalar que las puntuaciones deben ser calculadas mediante la sumatoria de los 12 ítems del instrumento final para obtener un índice general de percepción de la infidelidad en las redes sociales (excelente para estudiar correlaciones entre esta variable y otros constructos psicológicos). El orden de los ítems en la versión final fue aleatorio. Se entiende que, a mayor puntuación obtenida, mayor severidad en la clasificación de las conductas asociadas infidelidad en las redes sociales.

\section{Limitaciones y Fortalezas}

Al igual que toda investigación, nuestro estudio no está exento de limitaciones. Prime- ro, la muestra fue recopilada de forma accidental y no fue aleatoria; sin embargo, fue amplia y heterogénea. Segundo, no se pudo establecer la confiabilidad del instrumento a través del tiempo, solo se pudo hacer a través de sus elementos. Por último, el procedimiento para recoger los datos no fue estandarizado; esto puede afectar las medias del estudio y aumentar el error estándar de medición.

\section{Futuras Investigaciones}

Para futuras investigaciones se recomienda administrar la escala a otra muestra de participantes para realizar el proceso de validación cruzada. También sería un valor añadido examinar la confiabilidad temporal a través de la técnica de test-retest y realizar un análisis factorial confirmatorio. Se recomienda que el instrumento se valide en otras poblaciones latinoamericanas para auscultar sus propiedades psicométricas en distintos contextos nacionales e internacionales. Esto permitirá comparar el comportamiento de la escala en distintos contextos internacionales y facilitará estudiar la percepción de la infidelidad en las redes sociales desde una perspectiva multicultural.

En conclusión, las propiedades psicométricas obtenidas por la EPIRS demuestran índices de confiabilidad, de consistencia interna y validez dentro de lo esperado. Esto fue evidenciado al observar la consistencia interna del instrumento final y al examinar la construcción lógica del instrumento mediante un análisis factorial. Los análisis psicométricos demuestran que el instrumento es apropiado, por lo cual se recomienda su uso en futuras investigaciones en Puerto Rico que permitan fortalecer el conocimiento sobre las conductas asociadas a la infidelidad en las redes sociales y sus posibles correlaciones con otras 
variables sociales en Puerto Rico y el Caribe.

\section{Referencias}

Allan, G. (2004). Being unfaithful: His and her affairs. En J. Duncombe, K. Harrison, G. Allan \& D. Marsden (Eds.), The state of affairs: Explorations in infidelity and commitment (pp. 121-140). New Jersey, NJ: Erlbaum. doi: 10.4324/9781410610652-7

Allen, E. S., \& Baucom, D. H. (2006). Dating, marital, and hypothetical extradyadic involvements: How do they compare? Journal of Sex Research, 43(4), 307-317. doi: 10.1080/00224490609552330

Allens, E. S., Atkins, D. C., Baucom, D. H., Snyder, D. K., Gordon, K. C., \& Glass, S. P. (2005). Intrapersonal, interpersonal, and contextual factors in engaging in and responding to extramarital involvement. Clinical Psychology: Science and Practice, 12(2), 101-130. doi: 10.1093/clipsy.bpi014

Ato, M., López-García, J. J., \& Benavente, A. (2013). Un sistema de clasificación de los diseños de investigación en psicología. Anales de Psicología, 29(3), 1038-1059. doi: 10.6018/analesps.29.3.178511

Attrill, A. (2015). Cyberpsychology. Oxford, United Kingdom: Oxford University.

Becker, D. V., Sagarin, B. J., Guadagno, R. E., Millevoi, A., $\&$ Nicastle, L. D. (2004). When the sexes need not differ: Emotional responses to the sexual and emotional aspects of infidelity. Personal Relationships, 11(4), 529-538. doi: 10.1111/j.1475-6811.2004.00096.x

Berman, M. I., \& Frazier, P. A. (2005). Relationship power and betrayal experience as predictors of reactions to infidelity. Personality and Social Psychology Bulletin, 31(12), 1617-1627. doi: 10.1177/0146167205277209

Bernard, J. (1974). Infidelity: Some moral and social issues. En J. R. Smith \& L. G. Smith (Eds.), Beyond monogamy: Recent studies of sexual alternatives in marriage (pp. 138-158). Baltimore, MD: Johns Hopkins University.

Blow, A. J., \& Hartnett, K. (2005). Infidelity in commit- ted relationships II: A substantive review. Journal of Marital and Family Therapy, 31(2), 217-233. doi: 10.1111/j.1752-0606.2005.tb01556.x

Buunk, B. P. (1995). Sex, self-esteem, dependency and extradyadic sexual experience as related to jealousy responses. Journal of Social and Personal Relationships, 12(1), 147-153. doi: 10.1177/0265407595121011

Cano, A., \& O’Leary, K. D. (2000). Infidelity and separations precipitate major depressive episodes and symptoms of nonspecific depression and anxiety. Journal of Consulting and Clinical Psychology, 68(5), 774-781. doi: 10.1037//0022-006X.68.5.774

Comrey, A. L., \& Lee, H. B. (1992). A first course in factor analysis ( $2^{\mathrm{a}}$ ed.). Hillsdale, NJ: Lawrence Erlbaum.

DeVellis, R. (2017). Scale development: Theory and applications ( $4^{\mathrm{a}}$ ed.). Los Angeles, CA: Sage.

Docan-Morgan, T., \& Docan, C. A. (2007). Internet infidelity: Double standards and the differing views of women and men. Communication Quarterly, 55(3),317342. doi: 10.1080/01463370701492519

Field, A. P. (2013). Discovering statistics using IBM SPSS STATISTICS ( $4^{\mathrm{a}}$ ed.). London, England: SAGE.

Gackenbach, J., \& von Stackelberg, H. (2007). Self-Online: Personality and demographic implications. En J. Gackenbach (Ed.), Psychology and the Internet ( ${ }^{a}$ ed., pp. 55-73). Alberta, Canada: Academic. doi: 10.1016/B978-012369425-6/50022-9

Glass, S. P. (2002). Couple therapy after the trauma of infidelity. En A. S. Gurman \& N. S. Jacobson (Eds.), Clinical Handbook of Couple Therapy ( $3^{\mathrm{a}}$ ed., pp. 488-507). New York, NY: Guilford.

González-Galarza, J., Martínez-Taboas, A., \& Martínez-Ortiz, D. (2009). Factores psicológicos asociados a la infidelidad sexual y/o emocional y su relación a la búsqueda de sensaciones en parejas puertorriqueñas. Revista Puertorriqueña de Psicología, 20(1), 59-81. Recuperado de http://www.repsasppr.net/index.php/ reps

Hall, J. H., \& Fincham, F. D. (2006). Relationship dissolution following infidelity. En M. A. Fine \& J. H. Harvey (Eds.), Handbook of Divorce and Relationship 
Dissolution (pp. 153-168). Mahwah, NJ: Lawrence Erlbaum.

Hatcher, L. (1994). A step-by-step approach to using the SAS system for factor analysis and structural equation modeling. Cary, NC: SAS Institute.

IBM Corporation. (2016). IBM SPSS Statistics for Windows (Version 24.0) [Software de cómputo]. Armonk, NY: IBM.

Jung, S. (2013). Exploratory factor analysis with sma11 sample sizes: A comparison of three approaches. Behavioural Processes, 97, 90-95. doi: 10.1016/j. beproc.2012.11.016

Kline, P. (2000). Handbook of Psychological Testing. New York, NY: Routledge.

Kline, T. J. (2005). Psychological testing: A practical approach to design and evaluation. Thousand Oaks, CA: Sage. doi: 10.4135/9781483385693

Lawson, A. (1988). Adultery: An analysis of love and betrayal. Nueva York, NY: Basic Books.

Lloret-Segura, S., Ferreres-Traver, A., Hernández-Baeza, A., \& Tomás-Marco, I. (2014). El análisis factorial exploratorio de los ítems: Una guía práctica, revisada y actualizada. Anales de Psicología, 30(3), 11511169. doi: 10.6018/analesps.30.3.199361

Luo, S., Cartun, M. A., \& Snider, A. G. (2010). Assessing extradyadic behavior: A review, a new measure, and two new models. Personality and Individual Differences, 49(3), 155-163. doi: 10.1016/j. paid.2010.03.033

Mark, K., Janssen, E., \& Milhausen, R. (2011). Infidelity in heterosexual couples: Demographic, interpersonal, and personality-related predictors of extradyadic sex. Archives of Sexual Behavior, 40(5), 971-982. doi: 10.1007/s10508-011-9771-z

Montero, I., \& León, O. G. (2007). A guide for naming research studies in psychology. International Journal of Clinical and Health Psychology, 7(3), 847-862.

Munsch, C. L. (2015). Her support, his support: Money, masculinity and marital infidelity. American Sociological Review, 80(3), 469-495. doi: $10.1177 / 0003122415579989$
Pérez-Baquero, C. A., Ruiz-Santos, R. P., \& Parra-Ocampo, C. I. (2014). Efecto de una intervención cognitivo conductual en el conflicto marital por infidelidad. Psychologia: Avances de la Disciplina, 8(2), 23-36. doi: 10.21500/19002386.1219

Pittman, F. S., \& Wagers, T. P. (2005). The relationship, if any, between marriage and infidelity. Journal of Couple \& Relationship Therapy, 4(2-3), 135-148. doi: 10.1300/j398v04n02_12

Romero-Palencia, A. (2007). Infidelidad: Conceptualización, correlatos y predictores (Tesis doctoral). Universidad Nacional Autónoma de México (UNAM), México.

Schmitt, T. A. (2011). Current methodological considerations in exploratory and confirmatory factor analysis. Journal of Psychoeducational Assessment, 29(4), 304-321. doi: 10.1177/0734282911406653

Schneider, J. P. (2000). Effects of cybersex addiction on the family: Results of a survey. Sexual Addiction and Compulsivity, 7(1-2), 31-58. doi: 10.1080/10720160008400206

Shackelford, T. K., LeBlanc, G. J., \& Drass, E. (2000). Emotional reactions to infidelity. Cognition and Emotion, 14(5), 643-659. doi: 10.1080/02699930050117657

Stevens, J. (2002). Applied multivariate statistics for the social sciences ( $4^{\mathrm{a}}$ ed.). Mahwah, NJ: Lawrence Erlbaum.

Tafoya, M., \& Spitzberg, B. (2007). The dark side of infidelity: Its nature, prevalence, and communicative functions. En B. H. Spitzberg \& W. R. Cupach (Eds.), The dark side of interpersonal communication $\left(2^{\mathrm{a}}\right.$ ed., pp. 201-242). Mahwah, NJ: Lawrence Erlbaum. doi: 10.4324/9780203936849

Varela-Maceda, M. (2014). Estudio sobre infidelidad en la pareja: Análisis de contenido de la literatura. $\mathrm{Al}$ ternativas en Psicología, 30, 36-49. Recuperado de http://alternativas.me

Wasserman, M. (2015). Cyber Infidelity. Cape Town, Sudáfrica: Human \& Rousseau.

Whisman, M. A., \& Snyder, D. K. (2007). Sexual infidelity in a national survey of American women: Differen- 
ces in prevalence and correlates as a function of method of assessment. Journal of Family Psychology, 21(2), 147-154. doi: 10.1037/0893-3200.21.2.147

Wilson, F. R., Pan, W., \& Schumsky, D. A. (2012). Recalculation of the critical values for Lawshe's content validity ratio. Measurement and Evaluation in Counseling and Development, 45(3), 197-210. doi: 10.1177/0748175612440286

Wilson, K., Mattingly, B. A., Clark, E. M., Weidler, D. J., \& Bequette, A. W. (2011). The gray area: Exploring attitudes toward infidelity and the development of the perceptions of dating infidelity scale. Journal of Social Psychology, 151(1), 63-86. doi: 10.1080/00224540903366750 